\title{
Reconfigurable multi-standard uplink MIMO receiver with partial interference cancellation
}

\author{
Bei Yin*, Kiarash Amiri*, Joseph R. Cavallaro*, and Yuanbin Guo ${ }^{\dagger}$ \\ *ECE Department, Rice University, 6100 Main St., Houston, TX 77005 \\ Email: \{by2, kiaa, cavallar\}@ rice.edu \\ ${ }^{\dagger}$ US Wireless R\&D Center, Huawei Technologies, 1700 Alma Dr., Suite 500, Plano, TX 75002 \\ Email: yuanbinguo@huawei.com
}

\begin{abstract}
As HSPA/HSPA+ and LTE/LTE-A evolve in parallel, the reconfigurability of a receiver to support multiple standards has become more and more important, especially for small cells. In this paper, we first suggest a reconfigurable multistandard uplink MIMO receiver based on a frequency domain equalizer. Then, to improve the performance, we propose two low-complexity partial iterative interference cancellation (IC) schemes to deal with the residual inter-chip and inter-antenna interference in HSPA/HSPA+ and the residual inter-symbol and inter-antenna interference in LTE/LTE-A. Compared with a receiver consisting of separate HSPA/HSPA+ and LTE/LTE-A uplink receivers, this reconfigurable receiver can save up to 66.9\% complexity. Moreover, the two partial IC schemes have negligible performance loss compared with full IC scheme. They can achieve $2 \mathrm{~dB}$ gains in both standards with only $15.2 \%$ additional complexity to no IC scheme.
\end{abstract}

\section{INTRODUCTION}

LTE/LTE-A has been proposed to support up to $1 \mathrm{Gbps}$ in the downlink and $500 \mathrm{Mbps}$ in the uplink. At the same time, HSPA/HSPA+ is also being advocated to support up to 672 Mbps in the downlink and $70 \mathrm{Mbps}$ in the uplink. Because both standards coexist and evolve in parallel, it becomes more and more important for receivers to support multiple standards, while maintaining low complexity, especially for small cells.

After being introduced in [1], time domain equalizer (TDE) receivers have often been used in HSPA/HSPA+ base stations. On the other hand, frequency domain equalizer (FDE) receivers are used in LTE/LTE-A base stations. Simply putting HSPA/HSPA+ and LTE/LTE-A uplink receivers together results in a complicated receiver. The reason is that with the lack of commonality in TDE [1], [2] and FDE, it is hard to reduce the complexity of algorithm and architecture. In recent literature [3], [4], it was shown that FDE can be applied to the UMTS downlink without changing the current UMTS transmitter. In [3], the overlap-cut method is analyzed. In [4], the cyclic reconstruction method is presented. Both methods have good performance. However, compared with cyclic reconstruction, because overlap-cut does not need to perform matrix inversion, it has less complexity. By applying the idea in [3] to the HSPA/HSPA+ uplink and combining this receiver with a LTE/LTE-A uplink receiver, in this paper we suggest a reconfigurable multi-standard uplink MIMO receiver. Results show that the reconfigurable receiver has up to $66.9 \%$ less complexity than a receiver consisting of separate
HSPA/HSPA+ and LTE/LTE-A uplink receivers. This reduces the silicon area and power consumption.

In multi-tap channels, however, the residual interference of FDE degrades the performance. Interference cancellation (IC) can be used for mitigating this effect. Because of the spreading factor, IC needs to perform at the chip level in HSPA/HSPA+; while it needs to work at the symbol level in LTE/LTE-A. Recently, many IC schemes have been proposed, but only a few are for FDE and none is for multi-standard receivers. For example, in [5], all possible combinations of the current symbols are generated. For $N$ current symbols with BPSK, the receiver needs to list up to $2^{N}$ combinations, resulting in high complexity. In [6], the channel coefficients need to be sorted. This scheme also has high complexity. After being applied to FDE in [7]-[10], the IC scheme proposed in [11] no longer has low complexity. In [7], [8], IC is performed in the frequency domain for UMTS. This means extra DFTs are needed to convert the feedback signal to the frequency domain, which results in more complexity and significantly larger latency of the feedback signal. A similar issue occurs in LTE/LTE-A in [9], [10]. All above schemes either are too complicated or have large latency for real-time implementation.

In order to perform low-complexity IC for a multi-standard receiver, we therefore propose two partial iterative IC schemes. In HSPA/HSPA+, two schemes spread detected symbols into detected chips, regenerate interference from part of these chips, and perform inter-chip and inter-antenna IC. In LTE/LTE-A, these schemes regenerate interference from part of the detected symbols and perform inter-symbol and interantenna IC. Compared with other schemes, our schemes cancel only part of the interference instead of all of it. Furthermore, our IC does not need extra DFTs for the feedback signal, what result in less complexity and latency. Results show that our schemes have negligible performance loss compared with full IC, can bring up to $2 \mathrm{~dB}$ gain in both HSPA/HSPA+ and LTE/LTE-A, and only add $15.2 \%$ complexity to a reconfigurable multi-standard receiver without IC.

To the best of our knowledge, this is the first paper that analyzes reconfigurable multi-standard receivers and proposes IC for it. In section II, a reconfigurable multi-standard receiver is described. Section III presents our low-complexity partial iterative IC schemes. Simulation and complexity are shown in section IV and V. Section VI draws the conclusions. 


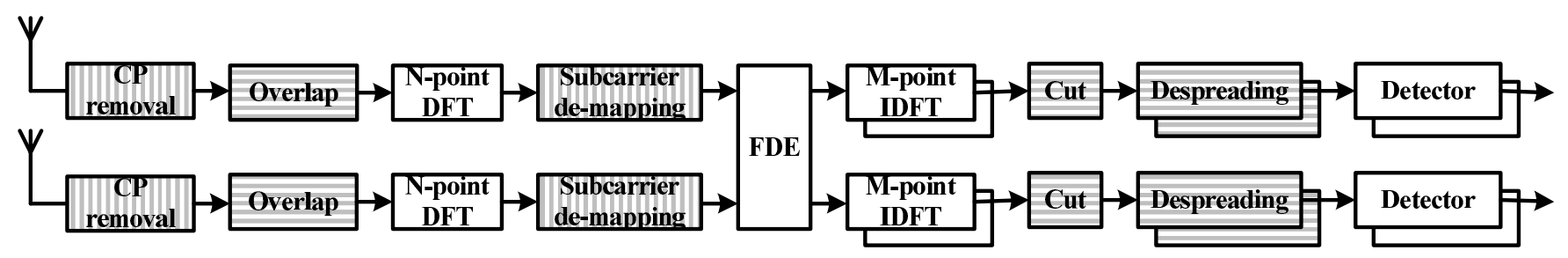

Fig. 1. Reconfigurable multi-standard uplink MIMO receiver (Horizontal shaded modules are unique to HSPA/HSPA+; Vertical shaded modules are unique to LTE/LTE-A; Non-shaded modules are shared by both standards).

\section{RECONFIGURABLE MULTI-STANDARD UPLINK MIMO RECEIVER}

A reconfigurable multi-standard receiver for an HSPA/HSPA+ and LTE/LTE-A uplink is shown in Fig. 1. The receiver is for a spatial multiplexing MIMO system with $N_{T}$ transmitter antennas and $N_{R}$ receiver antennas. The shaded modules marked by horizontal lines are unique to HSPA/HSPA+: overlap, cut, and despreading. The shaded modules marked by vertical lines are unique to LTE/LTE-A: cyclic prefix (CP) removal and subcarrier de-mapping. The non-shaded modules are shared by both standards.

\section{A. Receiver in HSPA/HSPA+}

Because there is no CP in HSPA/HSPA+, overlap-cut is used for helping apply FDE. This method is shown in Fig. 2. First, DFT is performed on the $N$ received chips. Next, after FDE, the $N$ equalized chips are converted to the time domain by IDFT. Finally, the middle $M$ chips are kept, and $(N-M) / 2$ chips from each side are discarded, because the middle section has less error than the edge regions.

By bypassing $\mathrm{CP}$ removal and subcarrier de-mapping modules, the receiver works in HSPA/HSPA+. The received chips at time $t$ are

$$
\mathbf{y}(t)=\sum_{i=0}^{L-1} \mathbf{h}(i) \mathbf{x}(t-i)+\mathbf{n}(t),
$$

where $\mathbf{y}(t)$ is an $N_{R} \times 1$ vector of received chips at time $t$; $\mathbf{h}(i)$ is an $N_{R} \times N_{T}$ time domain channel matrix at tap $i$ as

$$
\mathbf{h}(i)=\left(\begin{array}{ccc}
h_{1,1}(i) & \ldots & h_{1, N_{T}}(i) \\
\vdots & \ddots & \vdots \\
h_{N_{R}, 1}(i) & \ldots & h_{N_{R}, N_{T}}(i)
\end{array}\right) ;
$$

$L$ is the length of the channel; $\mathbf{x}(t-i)$ is an $N_{T} \times 1$ vector of transmitted chips at time $t-i ; \mathbf{n}(t)$ is an $N_{R} \times 1$ vector of additive white Gaussian noise with zero mean and variance $\sigma^{2}$ at time $t ; h_{n_{r}, n_{t}}(i)$ is the coefficient of the channel from transmitter antenna $n_{t}$ to receiver antenna $n_{r}$ at tap $i$.

Next, for each antenna, DFT converts $N$ chips selected by the overlap module to the frequency domain. The converted chips from all antennas are $\mathbf{Y}$.

By assuming the channel is known by the receiver, Minimum Mean Square Error (MMSE) FDE is applied to each frequency subcarrier by

$$
\mathbf{Y}_{e q}(n)=\left(\mathbf{H}(n)^{\mathrm{H}} \mathbf{H}(n)+\sigma^{2} \mathbf{I}\right)^{-1} \mathbf{H}(n)^{\mathrm{H}} \mathbf{Y}(n),
$$

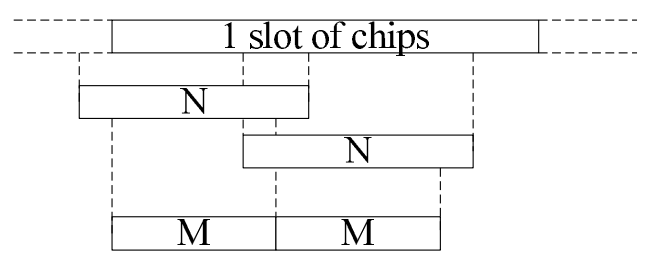

Fig. 2. Overlap-cut method for FDE.

where $\mathbf{Y}_{e q}(n)$ is an $N_{R} \times 1$ vector of equalized frequency domain chips on the $n$th subcarrier; $\mathbf{H}(n)$ is an $N_{R} \times N_{T}$ frequency domain channel matrix of the $n$th subcarrier; $\mathbf{Y}(n)$ is an $N_{R} \times 1$ vector of the frequency domain chips on the $n$th subcarrier; $n=1, \ldots, N$. An $N_{T} \times N_{T}$ equalized frequency domain channel matrix $\mathbf{H}_{e q}(n)$ is also computed by

$$
\mathbf{H}_{e q}(n)=\left(\mathbf{H}(n)^{\mathrm{H}} \mathbf{H}(n)+\sigma^{2} \mathbf{I}\right)^{-1} \mathbf{H}(n)^{\mathrm{H}} \mathbf{H}(n) .
$$

Then each IDFT converts $N$ equalized frequency domain chips and channel matrices to the time domain. The converted chips and channel matrices from all antennas are $\mathbf{y}_{e q}$ and $\mathbf{h}_{e q}$.

Later, the cut module for each antenna keeps the middle $M$ equalized time domain chips and discards $(N-M) / 2$ chips from each side. Finally, the kept chips are despread to symbols by the corresponding spreading factor of each user, and symbols are detected.

\section{B. Receiver in LTE/LTE-A}

Because of the existence of CP in LTE/LTE-A, FDE can be directly applied. By bypassing overlap, cut, and despreading modules, the receiver works in LTE/LTE-A. The flow is similar to HSPA/HSPA+.

First, symbols $\mathbf{y}(t)$ are received as in Eq. (1). Then CP is removed from $N+N_{C P}$ received symbols for each antenna, where $N_{C P}$ is the number of CP symbols. After that, DFT for each antenna converts $N$ kept symbols to the frequency domain. Next, frequency domain symbols are mapped to different users in the subcarrier de-mapping module, and MMSE-FDE is applied as in Eq. (3) to each frequency subcarrier. Finally, for each user, $M$ equalized frequency domain symbols are converted to the time domain by IDFT and then detected.

\section{Similarities and differences}

By using FDE, the reconfigurable multi-standard uplink MIMO receiver exploits the similarities between HSPA/HSPA+ and LTE/LTE-A uplink receivers. Modules having high complexity are shared: FDE, DFT, IDFT, and detector. 
This sharing results in much lower complexity. Although there are some unique modules for different standards, such as overlap, cut, despreading, CP removal, and subcarrier demapping modules, these modules have low complexity. These represent only a small percentage of the complexity of the entire receiver. The receiver complexity is shown in section V.

\section{LOW-COMPLEXITY PARTIAL ITERATIVE INTERFERENCE CANCELLATION}

In multi-tap channels, the residual interference still exists after FDE and degrades the performance. The problem becomes more complicated in a multi-standard receiver.

To solve this problem while keeping low complexity, we propose two partial iterative IC schemes: partial single-chip IC (SCIC) and partial multi-chip IC (MCIC). In HSPA/HSPA+, these schemes cancel inter-chip and inter-antenna interference, while in LTE/LTE-A they cancel inter-symbol and interantenna interference. Instead of fully cancelling the interference, our schemes cancel only part of it. As shown later, they have the same performance as full IC but with less complexity.

The schemes are shown in Fig. 3 and can be applied to the receiver in Fig. 1. The shaded modules marked by horizontal lines are unique to HSPA/HSPA+; non-shaded modules are common for both standards. Although our schemes work in both standards, they are discussed separately in the following sections to simplify the presentation.

\section{A. Interference cancellation in HSPA/HSPA+}

In HSPA/HSPA+, the equalized time domain chips are

$$
\mathbf{y}_{e q}(t)=\sum_{i=j-N+1}^{j} \mathbf{h}_{e q}(i) \mathbf{x}(t-i)+\mathbf{n}_{e q}(t),
$$

where $\mathbf{y}_{e q}(t)$ is an $N_{T} \times 1$ vector of equalized time domain chips at time $t ; j$ is equal to $(t \bmod N) ; \mathbf{h}_{e q}(i)$ is an $N_{T} \times N_{T}$ equalized time domain channel matrix at tap $i ; \mathbf{h}_{e q}(N+i)=$ $\mathbf{h}_{e q}(i) ; \mathbf{n}_{e q}(t)$ is an $N_{T} \times 1$ vector of equalized time domain additive white Gaussian noise at time $t$.

After the cut module, Eq. (5) can be written as

$$
\mathbf{y}_{e q}(t)=\mathbf{h}_{e q}(0) \mathbf{x}(t)+\sum_{i=1}^{M-1} \mathbf{h}_{e q}(i) \mathbf{x}(t-i)+\mathbf{n}_{e q}(t) .
$$

The first term is the transmitted chips. The second term is the residual interference from the other $(M-1) N_{T}$ previous chips at all antennas. This term can be minimized by regenerating the interference from $\mathbf{h}_{e q}(i)$ and the detected chips. To minimize the interference, two partial IC schemes are proposed.

1) Partial single-chip interference cancellation: When $N_{T}$ equalized time domain chips arrive, IC is performed by

$$
\begin{aligned}
\mathbf{y}_{i c}(t) & =\mathbf{f}\left(\mathbf{y}_{e q}(t)-\mathbf{y}_{r i}(t)\right) \\
& =\mathbf{f}\left(\mathbf{h}_{e q}(0) \mathbf{x}(t)+\mathbf{n}_{e q}(t)\right),
\end{aligned}
$$

where $\mathbf{y}_{i c}(t)$ is an $N_{T} \times 1$ vector of chips after IC at time $t ; \mathbf{f}$ is an $N_{T} \times N_{T}$ equalization matrix and will be defined later;

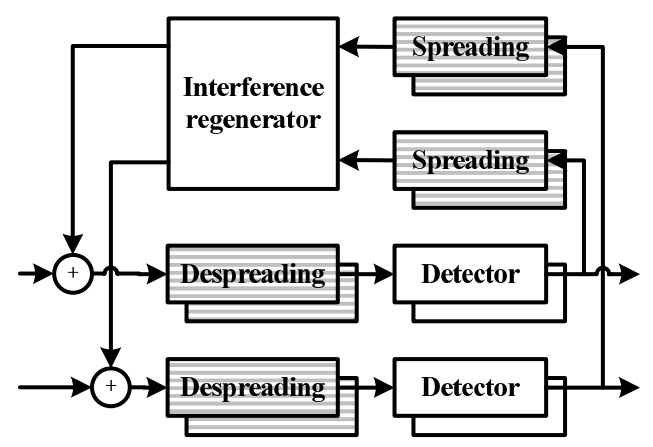

Fig. 3. Interference cancellation for multi-standard uplink MIMO receiver (Horizontal shaded unique to HSPA/HSPA+; Non-shaded shared by both).

$\mathbf{y}_{r i}(t)$ is an $N_{T} \times 1$ vector of regenerated interference at time $t$. The MMSE criterion is applied to $\mathbf{y}_{i c}(t)$ by choosing $\mathbf{f}$ as

$$
\mathbf{f}=\mathbf{h}_{e q}(0)^{\mathrm{H}}\left(\mathbf{h}_{e q}(0) \mathbf{h}_{e q}(0)^{\mathrm{H}}+\mathbf{C}_{\text {noise }}\right)^{-1},
$$

where $\mathbf{C}_{\text {noise }}$ is the covariance of the equalized noise $\mathbf{n}_{e q}(t)$,

$$
\mathbf{C}_{\text {noise }}=\mathbb{E}\left[\mathbf{n}_{e q}(t) \mathbf{n}_{e q}(t)^{\mathrm{H}}\right] .
$$

This can be calculated in the frequency domain by

$$
\mathbf{C}_{\text {noise }}=\frac{\sigma^{2}}{N} \sum_{n=1}^{N} \mathbf{A}(n) \mathbf{A}(n)^{\mathrm{H}},
$$

where $\mathbf{A}(n)$ is defined as

$$
\mathbf{A}(n)=\left(\mathbf{H}(n)^{\mathrm{H}} \mathbf{H}(n)+\sigma^{2} \mathbf{I}\right)^{-1} \mathbf{H}(n)^{\mathrm{H}} .
$$

As shown, because $\mathbf{A}(n)$ has already been calculated in Eq. (4), the computational complexity can be reduced.

Next, the despreading module of each user converts $\mathbf{y}_{i c}(t)$ together with the rest of chips in the same symbol into a single symbol. The equation is

$$
\mathbf{y}_{s}(p+1)=\frac{1}{L_{S F}} \sum_{i=1}^{L_{S F}} a(i) \mathbf{y}_{i c}\left(p L_{S F}+i\right),
$$

where $\mathbf{y}_{s}(p+1)$ is the $(p+1)$ th $N_{T} \times 1$ vector of symbols; $L_{S F}$ is the length of the spreading factor; $a(i)$ is the $i$ th element of the spreading factor. Each time, $N_{T}$ chips are despread to $N_{T} N_{U}$ symbols, where $N_{U}$ is the number of users.

Next, symbols are detected at each user. After that, the detected symbols of all users are spread to chips for IC. Each time, $N_{T} N_{U}$ detected symbols are spread to $N_{T}$ chips.

Finally, the interference regenerator produces interference from detected chips at all antennas by

$$
\mathbf{y}_{r i}(t)=\sum_{i=1}^{L_{F B}} \mathbf{h}_{e q}(i) \mathbf{c}(t-i),
$$

where $L_{F B}$ is the number of previous detected chips used in the interference regenerator; $\mathbf{c}(t-i)$ is an $N_{T} \times 1$ vector of detected chips at time $(t-i)$. When $L_{F B}=M-1$, the residual interference is regenerated from all previous $M-1$ chips, which is full regeneration. However, only the interference from neighbor chips is strong. The interference from other 
chips is weak. By adjusting $L_{F B}$ to only cover the strong interference, we can perform partial IC. The complexity can be much reduced without performance loss compared with full regeneration as shown in section IV. Moreover, because we use fewer detected chips for interference regeneration, less data needs to be stored.

2) Partial multi-chip interference cancellation: Because the despreading module converts the chip at time $t$ together with the chips in the same symbol to an undetected symbol, if the interference can be removed from all these chips, better performance can be achieved with more computation.

Based on this, partial MCIC is proposed. At time $t$, MCIC cancels the interference in all chips in the same symbol by

$$
\begin{aligned}
& \mathbf{y}_{i c}(t+j)=\mathbf{f}\left(\mathbf{y}_{e q}(t+j)-\mathbf{y}_{r i}(t+j)\right) \\
& =\mathbf{f}\left(\mathbf{h}_{e q}(0) \mathbf{x}(t+j)+\sum_{i=1}^{j} \mathbf{h}_{e q}(i) \mathbf{x}(t+j-i)+\mathbf{n}_{e q}(t+j)\right),
\end{aligned}
$$

where $j=0, \ldots,(p+1) L_{S F}-t$;

$$
\mathbf{y}_{r i}(t+j)=\sum_{i=j+1}^{L_{F B}} \mathbf{h}_{e q}(i) \mathbf{c}(t+j-i)
$$

After this, Eq. (12) performs the despreading.

\section{B. Interference cancellation in LTE/LTE-A}

In LTE/LTE-A, the equalized time domain symbols of each user have the same equation as Eq. (5). However, without the cut module, Eq. (5) is directly rewritten as

$$
\mathbf{y}_{e q}(t)=\mathbf{h}_{e q}(0) \mathbf{x}(t)+\sum_{\substack{i=j-M+1 \\ i \neq 0}}^{j} \mathbf{h}_{e q}(i) \mathbf{x}(t-i)+\mathbf{n}_{e q}(t),
$$

where $\mathbf{y}_{e q}(t)$ is an $N_{T} \times 1$ vector of equalized time domain symbols at time $t ; j$ is equal to $(t \bmod M) ; \mathbf{h}_{e q}(i)$ is an $N_{T} \times N_{T}$ equalized time domain channel matrix at tap $i$; $\mathbf{h}_{e q}(M+i)=\mathbf{h}_{e q}(i) ; \mathbf{n}_{e q}(t)$ is an $N_{T} \times 1$ vector of equalized time domain additive white Gaussian noise at time $t$. The first term in the equation is the transmitted symbols. The second term is the residual interference from the other $(M-1) N_{T}$ symbols in the same IDFTs at all antennas. This term can be minimized by regenerating the interference from $\mathbf{h}_{e q}(i)$ and detected symbols. Because there is no spreading factor, the two IC schemes in HSPA/HSPA+ become a single equivalent partial IC in LTE/LTE-A.

When $N_{T}$ equalized time domain symbols of each user arrive, IC is performed by Eq. (7). Next, without despreading, the symbols are directly detected.

Finally, the interference regenerator produces interference from the detected symbols at all antennas by

$$
\mathbf{y}_{r i}(t)=\sum_{\substack{i=-L_{F F} \\ i \neq 0}}^{L_{F B}} \mathbf{h}_{e q}(i) \mathbf{s}(t-i)
$$

where $L_{F F}$ is the number of future detected symbols used in the interference regenerator. However, these symbols are not received in the future, but already received and buffered. When $L_{F B}+L_{F F}=M-1$, the residual interference is regenerated from all other $M-1$ symbols in the same IDFT, which is full regeneration. As in HSPA/HSPA+, by adjusting $L_{F B}+L_{F F}$ to only cover the strong interference, the complexity can be reduced without performance loss, and less storage is needed.

\section{Similarities and differences}

As described, the flow of partial IC in HSPA/HSPA+ is the same as in LTE/LTE-A. Although the interference regeneration in Eq. (17) looks different from Eq. (13), Eq. (17) becomes Eq. (13) when $L_{F F}=0$. Thus, same interference regenerator can be reconfigured to support both standards.

\section{Simulations}

Several schemes are compared in this section. They are:

1) No IC: There is no IC.

2) Parallel FD IC: All symbols are first detected, and then IC is performed in the frequency domain by using all detected symbols, similar to the schemes in [7]-[10].

3) Full IC: Symbol detection and IC perform iteratively with $L_{F B}=M-1$ in Eq. (13).

4) Partial IC: These are our proposed schemes. Symbol detection and IC perform iteratively with $L_{F B}<M-1$.

5) Perfect IC: Similar to full IC, except that accurate transmitted symbols are used as detected symbols.

The simulation parameters are defined in Table I. They are chosen according to HSPA/HSPA+ and LTE/LTE-A standards. Orthogonal variable spreading factor (OVSF) is configured to support the highest data rate for E-DPDCH in HSPA/HSPA+.

TABLE I

SIMULATION PARAMETERS FOR 5 MHZ CHANNEL BANDWIDTH

\begin{tabular}{|c|c|}
\hline Parameter & Value \\
\hline \hline Rayleigh channels & {$[0-4.7712-7.7815-7.7815] \mathrm{dB}$} \\
\hline Length of DFT & 512 \\
\hline Length of IDFT & 512 (HSPA/HSPA+); 300 (LTE/LTE-A) \\
\hline Length of CP & 36 (LTE/LTE-A) \\
\hline Modulation order & $16-\mathrm{QAM}$ \\
\hline Number of antennas & $4 \times 4$ \\
\hline OVSF & {$[224$ (HSPA/HSPA+) } \\
\hline FDE & MMSE-FDE \\
\hline$L_{F F}$ & 15 (LTE/LTE-A) \\
\hline$L_{F B}$ & 15 \\
\hline
\end{tabular}

The performance in HSPA/HSPA+ is shown Fig. 4. Our partial IC schemes can improve the performance of no IC up to $2 \mathrm{~dB}$. They also have $0.5 \mathrm{~dB}$ gain over parallel $\mathrm{FD}$ IC. This is because that different from parallel IC, partial IC performs detection and IC iteratively, so later IC is performed on more accurate detected symbols. The figure shows that our schemes have negligible performance loss compared with full IC, and have close performance to perfect IC. This means that cancelling only the strong interference does not degrade the performance. The figure also shows that the performance of 


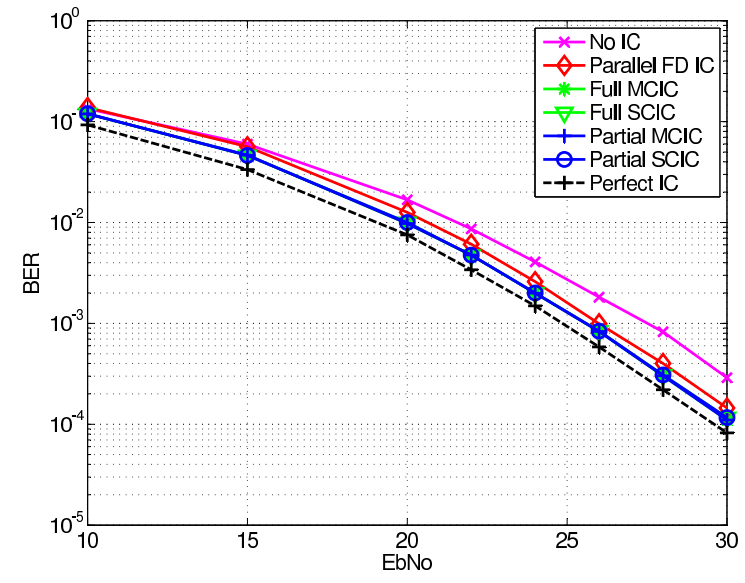

Fig. 4. Performance in HSPA/HSPA+ with $4 \times 4$ MIMO in Rayleigh channels.

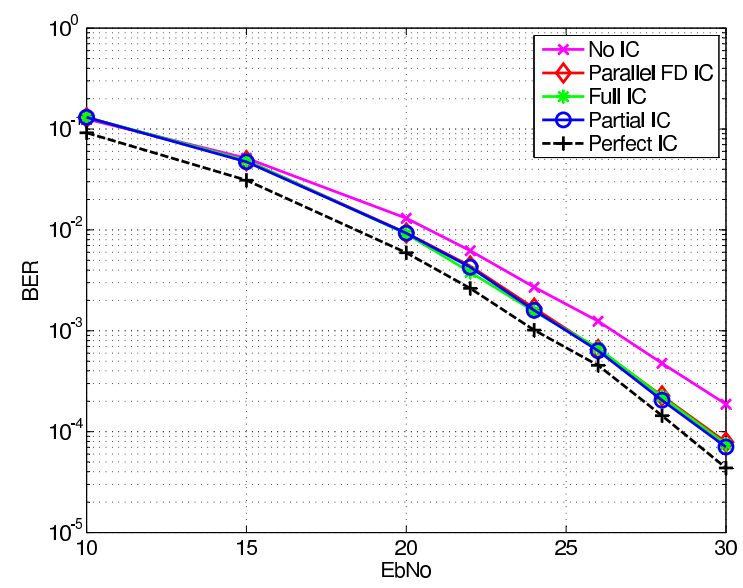

Fig. 5. Performance in LTE/LTE-A with $4 \times 4$ MIMO in Rayleigh channels.

partial MCIC is slightly better than partial SCIC, while as mentioned in section III, partial SCIC has lower complexity than partial MCIC.

The performance improvement in LTE/LTE-A, shown in Fig. 5, is similar to that in HSPA/HSPA+. This means that our scheme works well in both standards.

\section{COMPLEXITY ANALYSIS}

The complexity of receivers is shown in Table II. Parameters are chosen as shown in Table I. The complexity is represented by the number of equivalent multiplications needed by 512 MIMO chips/symbols in HSPA/HSPA+ or LTE/LTE-A.

The ratiol in the table indicates that when the size of MIMO increases, a reconfigurable receiver saves more complexity from a receiver with separate HSPA/HSPA+ and LTE/LTEA uplink receivers. The table also shows that our partial IC can reduce the complexity of parallel FD IC by $80.5 \%$.

\section{CONCLUSION}

In this paper, we suggest a reconfigurable multi-standard uplink MIMO receiver to support both HSPA/HSPA+ and
TABLE II

NUMBER OF EQUIVALENT MULTIPLICATION FOR 512 MIMO CHIPS/SYMBOLS

\begin{tabular}{|c|c|c|c|}
\hline Number of antennas & $\mathbf{1} \times \mathbf{1}$ & $\mathbf{2} \times \mathbf{2}$ & $\mathbf{4} \times \mathbf{4}$ \\
\hline \hline Reconfigurable multi-standard receiver & 7150 & 14319 & 28791 \\
\hline HSPA/HSPA+ \& LTE/LTE-A receivers & 7671 & 22994 & 87037 \\
\hline Ratio1 & $\mathbf{9 3 . 3 \%}$ & $\mathbf{6 2 . 3 \%}$ & $\mathbf{3 3 . 1 \%}$ \\
\hline \hline Partial IC & 255 & 1045 & 4370 \\
\hline Parallel FD IC & 3794 & 8788 & 22376 \\
\hline Ratio2 = Partial IC/Parallel FD IC & $\mathbf{6 . 7 \%}$ & $\mathbf{1 1 . 9 \%}$ & $\mathbf{1 9 . 5 \%}$ \\
\hline Ratio3 = Partial IC/Reconfigurable receiver & $\mathbf{3 . 6 \%}$ & $\mathbf{7 . 3 \%}$ & $\mathbf{1 5 . 2 \%}$ \\
\hline
\end{tabular}

LTE/LTE-A. Analysis shows that this reconfigurable receiver can save up to $66.9 \%$ complexity. We further propose two lowcomplexity partial iterative IC schemes to reduce the residual interference of the reconfigurable receiver. Results show that our schemes can bring up to $2 \mathrm{~dB}$ gain in both standards as full IC performs, but with only $15.2 \%$ additional complexity.

\section{ACKNOWLEDGMENT}

This work was supported in part by Renesas Mobile, Texas Instruments, Xilinx, Samsung, Huawei, and US National Science Foundation under grants EECS-0925942 and CNS0923479.

\section{REFERENCES}

[1] G. Bottomley, T. Ottosson, and Y.-P. Wang, "A generalized RAKE receiver for interference suppression," IEEE Journal on Selected Areas in Communications, vol. 18, no. 8, pp. 1536 -1545, Aug. 2000.

[2] Y. Guo, J. Zhang, D. McCain, and J. Cavallaro, "An Efficient Circulant MIMO Equalizer for CDMA Downlink: Algorithm and VLSI Architecture," EURASIP Journal on Applied Signal Processing, Feb. 2006.

[3] L. Martoyo, T. Weiss, F. Capar, and F. Jondral, "Low complexity CDMA downlink receiver based on frequency domain equalization," in IEEE 58th Vehicular Technology Conference, 2003. VTC 2003-Fall. 2003, vol. 2, Oct. 2003, pp. $987-991$ Vol.2.

[4] Y. Li, S. McLaughlin, and D. Cruickshank, "UMTS FDD frequency domain equalization based on self cyclic reconstruction," in IEEE International Conference on Communications, 2005. ICC 2005. 2005, vol. 3, May 2005, pp. $2122-2126$ Vol. 3.

[5] J. Yang and Y. Li, "Tentative chip decision-feedback equalizer for multicode wideband CDMA," IEEE Transactions on Wireless Coтmиnications, vol. 4, no. 1, pp. 137 - 148, Jan. 2005.

[6] M. Melvasalo, P. Janis, and V. Koivunen, "MMSE equalizer and chip level inter-antenna interference canceler for HSDPA MIMO systems," in IEEE 63rd Vehicular Technology Conference, 2006. VTC 2006-Spring., vol. 4, May 2006, pp. $2008-2012$.

[7] L. Ren, G. Xu, and L. Sang, "An Improved Frequency-Domain Interference Cancellation with DFE for CDMA," in IEEE 68th Vehicular Technology Conference, 2008. VTC 2008-Fall., Sep. 2008, pp. 1 -4.

[8] K. Takeda and F. Adachi, "Frequency-Domain Interchip Interference Cancelation for DS-CDMA Downlink Transmission," IEEE Transactions on Vehicular Technology, vol. 56, no. 3, pp. 1286 -1294, May 2007.

[9] S. Okuyama, K. Takeda, and F. Adachi, "Iterative MMSE Detection and Interference Cancellation for Uplink SC-FDMA MIMO Using HARQ," in IEEE International Conference on Communications (ICC), 2011, Jun. 2011, pp. $1-5$.

[10] M. Jiang, N. Prasad, and X. Wang, "Design of high performance MIMO receivers for LTE/LTE-A uplink," in Signals, Systems and Computers (ASILOMAR), 2010 Conference Record of the Forty Fourth Asilomar Conference on, Nov. 2010, pp. $1493-1497$.

[11] X. Wang and H. Poor, "Iterative (turbo) soft interference cancellation and decoding for coded CDMA," IEEE Transactions on Communications, vol. 47, no. 7, pp. $1046-1061$, Jul. 1999. 